\title{
The Influence of Kinesiophobia on Perceived Disability in Patients With an Upper-Extremity Injury: A Critically Appraised Topic
}

\author{
Olivia Bartlett and James L. Farnsworth II
}

\begin{abstract}
Clinical Scenario: Kinesiophobia is a common psychological phenomenon that occurs following injury involving fear of movement. These psychological factors contribute to the variability among patients' perceived disability scores following injury. In addition, the psychophysiological, behavioral, and cognitive factors of kinesiophobia have been shown to be predictive of a patient's self-reported disability and pain. Previous kinesiophobia research has mostly focused on lower-extremity injuries. There are fewer studies that investigate upper-extremity injuries despite the influence that upper-extremity injuries can have on an individual's activities of daily living and, therefore, disability scores. The lack of research calls for a critical evaluation and appraisal of available evidence regarding kinesiophobia and its contribution to perceived disability for the upper-extremity. Focused Clinical Question: How does kinesiophobia in patients with upper-extremity injuries influence perceptions of disability and quality of life measurements? Summary of Key Findings: Two cross-sectional studies and one cohort study were included. The first study found a positive relationship between kinesiophobia and a high degree of perceived disability. Another study found that kinesiophobia and catastrophic thinking scores were the most important predictors of perceived upper-extremity disability. The third study found that kinesiophobia contributes to self-reported disability in the shoulder. Clinical Bottom Line: There is moderate evidence that supports the relationship between kinesiophobia and perceived disability, and the relationship between elevated perceptions of disability and increased kinesiophobia scores in patients with an upper-extremity injury. Clinicians should evaluate and monitor kinesiophobia in patients following injury, a condition that can enhance perceptions of disability. An elevated perception of disability can create a cycle of fear that leads to hypervigilance and fear-avoidance behavior. Strength of Recommendation: Consistent findings from reviewed studies suggest there is grade B evidence to support that kinesiophobia is related to an increased perceived disability following upper-extremity injuries.
\end{abstract}

Keywords: fear of movement, catastrophizing, pain-related anxiety

\section{Clinical Scenario}

It has been well documented in the literature that having an injury can significantly impact an individual's psychological state. ${ }^{1}$ The Fear-Avoidance Model, a cognitive-behavioral model, provides a premise that the experience of pain can be interpreted in one of 2 ways. On one hand, an individual can view the pain as nonthreatening and will likely continue to engage in activities of daily living. ${ }^{2}$ On the other hand, pain catastrophizing, or dwelling on the most negative consequences, can occur. ${ }^{2}$ Pain catastrophizing will likely result in kinesiophobia, hypervigilance through heightened alertness to body sensations, and safety-seeking behaviors. ${ }^{2}$ It appears that negative psychological responses, such as kinesiophobia, can significantly affect the quality and length of the patient's rehabilitation time. ${ }^{1}$ Furthermore, previous research has shown that psychological morbidity can predict injury outcomes including pain and physical function. ${ }^{3}$

Kinesiophobia is a common psychological phenomenon that involves fear of movement that can occur following injury. ${ }^{4}$ This concept is a component of the Fear-Avoidance Model that encompasses several psychological factors (eg, pain catastrophizing, depression, and hypervigilance) that can contribute to the development of disability and chronic conditions. ${ }^{5}$ Kinesiophobia

Bartlett is with the University of Illinois Springfield, Springfield, IL, USA. Farnsworth is with the Department of Health and Human Performance, Texas State University, San Marcos, TX, USA. Farnsworth (farnsworth@txstate.edu) is corresponding author. can be classified into 2 different subcategories: a somatic focus or an activity avoidance focus. ${ }^{4} \mathrm{~A}$ somatic focus is the belief that pain represents a serious underlying medical condition, whereas an activity avoidance focus is the belief that movement can result in further injury. ${ }^{4}$ These psychological factors have been shown to contribute to the differences between individuals' disability perceptions following an injury, as well as predict disability following an injury. ${ }^{4}$ Kinesiophobia is typically evaluated and measured using a self-reported patient-outcomes measure, such as the Tampa Scale of Kinesiophobia-11 (TSK-11). ${ }^{6}$ It is important to note that kinesiophobia should not be viewed as a dichotomous category ${ }^{4}$ Rather, kinesiophobia has various levels of severity and should be looked at on a spectrum. ${ }^{4}$ Use of these scales in the clinical setting is important because they allow for evaluation of kinesiophobia as a spectrum, rather than a dichotomous diagnosis.

Kinesiophobia can begin to influence an individual's activities of daily living and quality of life due to fear of performing daily tasks. ${ }^{7}$ This perpetuates a cycle of fear leading to hypervigilance, which leads to prolonged recovery time and increased chronic pain. ${ }^{8}$ Following an injury, kinesiophobia can cause activity avoidance behavior that leads to hypervigilance of movement, work, and activities of daily living. This behavior can eventually worsen the disability and elevate the development of chronic pain. ${ }^{8}$ Previous research has stated that fear of injury or reinjury impacts performance through diminished concentration and confidence, which ultimately increases the risk of actual reinjury. ${ }^{9}$ In addition, lack of trust in the injured site may be a factor that leads to reinjury or the development of chronic disability or pain. ${ }^{8,9}$ It is important that 
clinicians and health care professionals are knowledgeable in the recognition, evaluation, and treatment of patients with kinesiophobia. By improving the knowledge of the effect of kinesiophobia on patient perceptions of disability, clinicians can help improve care and reduce the kinesiophobia that impedes recovery time. ${ }^{6}$ Clinicians can also include addressing kinesiophobia as one of the patient's rehabilitation goals and provide intervention throughout their treatment. ${ }^{6}$

The effect of kinesiophobia on patient perceptions of disability has been examined in patients with low back pain, ${ }^{4}$ neck pain, ${ }^{10}$ and knee injury ${ }^{11}$; however, there is a smaller body of research pertaining to the effects of kinesiophobia in patients with upperextremity injuries. Upper-extremity injuries can negatively impact activities of daily living (eg, driving, changing clothes, reaching), leading to diminished quality of life and overall well-being. ${ }^{7}$ The substantial influence that the upper-extremity has on an individual's activities of daily living calls for more investigation into the relationship between kinesiophobia and upper-extremity injuries. This critically appraised topic was conducted to evaluate and appraise the available evidence regarding the relationship between kinesiophobia and patient perceptions of disability in upperextremity injury.

\section{Focused Clinical Question}

In patients with upper-extremity injuries, how does kinesiophobia influence perceptions of disability and quality of life measurements?

\section{Search Strategy}

\section{Terms Used to Guide Search Strategy}

- Patient/Client Group: patients with a traumatic or nontraumatic upper-extremity injury

- Intervention/Assessment: Tampa Scale of Kinesiophobia

- Comparison: patient perceptions of disability

- Outcomes: kinesiophobia

\section{Sources of Evidence Searched}

- EBSCOhost

- PsycINFO

- CINAHL

- Additional resources obtained via reference lists

\section{Summary of Search, "Best Evidence" Appraised, and Key Findings}

- The literature was searched for studies of level 3 evidence or higher that investigated the relationship between kinesiophobia and patient perceptions of disability in upper-extremity injuries.

- The search of the literature yielded 29 articles. A total of 3 studies were included that fully matched the inclusion and exclusion criteria established prior to the search. Two of the studies were cross-sectional designs, and one was a prospective cohort design.
- Results of the search provided moderate evidence to support the relationship between kinesiophobia and perceived disability.

- Feleus et $\mathrm{al}^{10}$ investigated the relationship between kinesiophobia and upper-extremity injuries among 679 general practice patients (ages 18-64 y). Participants completed the TSK-AV and other questionnaires a total of 3 times over the course of 12 months. The mean TSK-AV score was calculated to assess the variables for the total population, and a multivariate regression analysis was conducted to assess the contributing variables. Authors found a positive relationship between TSK scores and pain catastrophizing, a high degree of disability, and comorbid musculoskeletal complaints. ${ }^{10}$

- De et $\mathrm{al}^{4}$ investigated the relationship between kinesiophobia, catastrophic thinking, and upper-extremity injury among 319 patients receiving treatment for various upper-extremity injuries. Participants completed the TSK and other questionnaires that measured depression, pain anxiety, catastrophic thinking, kinesiophobia, and perceived level of support. A Pearson correlation and an analysis of variance were used to assess the variables. Authors found that kinesiophobia/catastrophic thinking scores were the most important independent predictors of upper-extremity disability. ${ }^{4}$

- Lentz et $\mathrm{al}^{8}$ investigated the relationship between pain intensity, physical impairment, and kinesiophobia among 142 patients with nonsurgical, unilateral shoulder pain. Participants completed the TSK-11 and a clinical shoulder evaluation. A Pearson correlation and a regression model were used to assess the variables. Authors found that average pain intensity and shoulder-flexion range of motion were the largest contributors to perceived disability. ${ }^{8}$

- All of the included studies found that kinesiophobia scores are significantly influenced by catastrophic thinking or perceived disability in the upper-extremity.

- Other variables that were assessed and found to influence kinesiophobia scores included social support, depression, pain anxiety, and pain intensity.

\section{Clinical Bottom Line}

Clinicians should evaluate and monitor kinesiophobia in patients following an upper-extremity injury, a condition that can lead to enhanced perceptions of disability. Increased levels of kinesiophobia in patients with upper-extremity injuries may influence part of the variance associated with increased perceptions of disability and lead to decreased health-related quality of life.

\section{Strength of Recommendation}

Based on the consistent evidence from the Centre for EvidenceBased Medicine level 3 studies included in this critically appraised topic, there is grade B Strength of Recommendation Taxonomy ${ }^{12}$ evidence supporting the relationship between kinesiophobia and perceived disability.

\section{Inclusion and Exclusion Criteria}

The criteria for study inclusion were as follows:

- Studies that included participants with an upper-extremity injury

- Studies that included perceived disability 
- Studies using a patient-reported outcome measure to assess kinesiophobia.

The criteria for study exclusion were as follows:

- Studies that did not include participants with an upper-extremity injury

- Studies that did not include perceived disability

- Studies that did not use a patient-reported outcome measure to assess kinesiophobia.

\section{Results of Search}

Three studies which met the inclusion criteria and are categorized based on the 2011 Centre of Evidence-Based Medicine Levels of Evidence have been listed in Table 1.

\section{Best Evidence}

The 3 studies in Table 1 were identified as the best evidence available and selected for inclusion in this critically appraised topic. Table 2 describes these studies, which best fit the inclusion criteria and investigated the relationship between kinesiophobia and patient perceptions of disability in those with upper-extremity injuries. While there is a lack of randomized controlled trials on this topic, the methods and assessments in each study led to strong and accurate results.

\section{Implications for Practice, Education, and Future Research}

The goal of this critically appraised topic was to review current evidence regarding the relationship between kinesiophobia and patient perceptions of disability in those with upper-extremity injuries. There is currently moderate evidence regarding the psychological effect of kinesiophobia on self-reported disability in patients with upper-extremity injuries. All 3 of the appraised studies found that either pain catastrophizing, kinesiophobia, or pain-related fear impacted the perception of disability in the upperextremity. ${ }^{4,8,10}$ All reviewed studies were characterized as level 3 evidence according to the Oxford Centre of Evidence-Based Medicine. Based on the findings in this critically appraised topic, it can be concluded that clinicians should be evaluating and monitoring kinesiophobia in patients following an upper-extremity injury.

While these articles have moderate evidence supporting a psychological impact of kinesiophobia, some differences were found between studies that were included. Both Feleus et $\mathrm{al}^{10}$ and De et $\mathrm{al}^{4}$ found that pain catastrophizing was the strongest contributor to perceived disability scores in patients with upperextremity injuries. However, each study analyzed other variables that made smaller contributions to average perceived disability scores. For example, Feleus et $\mathrm{al}^{10}$ found that perceived disability and comorbid musculoskeletal complaints also significantly impacted TSK scores. De et $\mathrm{al}^{4}$ concluded that kinesiophobia was also one of the strongest influences on Disability of Arm Shoulder and Hand scores. On the contrary, Lentz et $\mathrm{al}^{8}$ concluded that average pain intensity and shoulder-flexion range of motion were the strongest contributors to Shoulder Pain and Disability Index scores. However, these authors noted that they did not assess pain catastrophizing as a contributing factor to Shoulder Pain and Disability Index scores. This could explain the difference in findings compared with the other studies.

These studies were not without limitations. One limitation was that each study failed to include at least one component of the FearAvoidance Model in their study. Specifically, pain catastrophizing, depression, or hypervigilance were left out of some studies. Another limitation of this study was that the studies included in this critically appraised topic also did not examine any variance related specifically to kinesiophobia. This could indicate that there are other contributing and compounding factors that may influence the perceived disability found in these studies. The included studies also evaluated variables such as pain-anxiety, social support, depression, catastrophic thinking, and so on. These variables could be considered factors that may have contributed to the variance in kinesiophobia scores. In addition, there was a lack of participant demographic information. The limited participant demographic information could have also contributed to the factors that influenced variance in kinesiophobia scores across these studies. All 3 studies used linear regression models to evaluate the significance of predictor variables on kinesiophobia; however, the selection of which variables to include in the models initially was based upon the strength of univariate correlations between kinesiophobia scales and other outcome measures. Because each of these studies included different outcomes, this could have led to differences in interpretation of effect for some variables. Also, it is important to add that although all 3 studies used regression models, each study used a different reduction method. For example, Lentz et $\mathrm{al}^{8}$ used a hierarchical regression model, where variables are placed sequentially into the model based upon theory, while Feleus et $\mathrm{al}^{10}$ and De et $\mathrm{al}^{4}$ used stepwise regression models, where the variables are included in the model sequentially based upon statistical significance alone. Another limitation could be various research methods between the included studies. The study by Feleus et $\mathrm{al}^{10}$ conducted a follow-up evaluation on their participants but excluded the follow-up findings from their results. Failure to include the follow-up findings eliminates the ability to assess kinesiophobia over time. Tracking if, and how, kinesiophobia changed over time would have been valuable information for clinicians. Finally, the included studies focus primarily on the general population. This creates strong generalizability for the nonathletic population but excludes the applicability to the athletic populations, who are at a high risk for upper-extremity injuries. Future research should investigate the magnitude that kinesiophobia has on patient perception of disability. In addition, future research should investigate how clinicians can implement strategies to prevent or minimize kinesiophobia among their patients.

Table 1 Summary of Study Designs

\begin{tabular}{|c|c|c|c|}
\hline Level of evidence & Study design & Number located & Reference \\
\hline \multirow[t]{2}{*}{3} & Cross-sectional & 2 & $\begin{array}{l}\text { Lentz et } \mathrm{al}^{8} \\
\text { De et } \mathrm{al}^{4}\end{array}$ \\
\hline & Prospective cohort & 1 & Feleus et $\mathrm{al}^{10}$ \\
\hline
\end{tabular}


Table 2 Characteristics of Included Studies

\begin{tabular}{ll}
\hline $\begin{array}{l}\text { Study } \\
\text { characteristics }\end{array}$ & Feleus et al $^{\mathbf{1 0}}$ \\
\hline Design & Prospective cohort \\
Participants & 679 participants (age 18-64 y; males = \\
& 281 and females = 398) who visited a \\
& $\begin{array}{l}\text { general practitioner with a new complaint } \\
\text { or new episode of a complaint associated } \\
\text { with the upper-extremity. }\end{array}$
\end{tabular}

Intervention investigated

Outcome measure(s)

Main findings

CEBM level of 3

evidence

STROBE score

Conclusion of TSK-AV, DASH, 4DSQ, SOS, and the CSQ during their initial visit and again at 6 and 12 mo follow-up.

Changes in kinesiophobia, social support, catastrophizing, somatization, and distress over time.

Multivariate linear regression revealed significant associations between kinesiophobia and self-perceived disability, degree of catastrophizing, degree of disability, comorbidity of musculoskeletal complaints, having a shoulder-specific complaint, social support, somatization, and distress. All variables were significant at the .05 level.
Adults with a high degree of catastro-

Cross-sectional

142 participants (mean age 41.4 [18.5] y; males $=78$ and females $=64$ ) with nonsurgical unilateral shoulder pain were recruited for this study.
Lentz et $\mathrm{al}^{8}$

Participants completed a modified version
Participants completed a demographic questionnaire reporting information and pain intensity; SPADI; the shortened TSK-11; and a clinical assessment for shoulder ROM.

Kinesiophobia score, SPADI function subscore, and SPADI pain score.

SPADI function subscale was significantly associated with TSK-11 scores $(r=.288, P=.001)$, average pain intensity $(r=.380, P<.001)$, and flexion ROM index $(r=.45, P<.001)$. The results of the multiple regression analysis reported that average pain intensity and flexion ROM contributed to $22 \%(P<.001)$ of the variance of shoulder disability. In addition, TSK-11 scores contributed 3\% $(P<.001)$ of variance of shoulder disability.

3

$19 / 22$

Fear of pain contributed a small amount to self-reported disability in patients with nonoperative unilateral shoulder pain. related to injury mechanism, symptoms,

De et $\mathrm{al}^{4}$

Cross-sectional

A total of 319 (males $=137$ and females $=182$ ) participants receiving treatment for one of the following conditions: trigger finger $(n=94)$, carpal tunnel syndrome $(n=29)$, trapeziometacarpal arthrosis $(\mathrm{n}=33)$, Dupuytren contracture $(n=31)$, de Quervain syndrome $(n=28)$, wrist ganglion cyst $(n=32)$, lateral epicondylosis $(n=41)$, or a nonoperative distal radial fracture $(n=31)$.

Participants were asked to complete a battery of self-reported questionnaires including the DASH, CES-D, PCS, TSK, PASS-20, and WHYMPI.

Shoulder disability, depressive symptoms, catastrophic thinking, kinesiophobia, pain-related anxiety, and perceived partner support.

DASH scores were significantly associated with depression $(r=.39, P<.01)$, catastrophic thinking $(r=.57, P<.01)$, kinesiophobia $(r=.47, P<.01)$, and pain anxiety $(r=.30, P<.01)$. The stepwise regression analysis reported that sex, diagnosis, employment status, kinesiophobia, and catastrophic thinking accounted for $55 \%$ of variability of DASH scores. Catastrophic thinking (partial $R^{2}=17.7 \%$ ) and kinesiophobia (partial $R^{2}=6.3 \%$ ) are the most important predictors of upper-extremity disability. 3

$19 / 22$ phizing, disability, comorbidity of musculoskeletal complains, low self-support, high somatization, high distress, or with a shoulder-related injury are likely to report higher levels of kinesiophobia.
Catastrophic thinking and kinesiophobia accounted for disability in those with upper-extremity injury.

\footnotetext{
Abbreviations: 4DSQ, 4-Dimensional Symptom Questionnaire; CEBM, Centre for Evidence-Based Medicine; CES-D, Center for Epidemiologic Studies Depression Scale; CSQ, Coping Strategy Questionnaire; DASH, Disability of Arm Shoulder and Hand; PASS-20, Pain Anxiety Symptoms Scale; PCS, Pain Catastrophizing Scale; ROM, range of motion; SOS, Social Support Scale; SPADI, Shoulder Pain and Disability Index; STROBE, Strengthening the Reporting of Observational Studies in Epidemiology; TSK-AV, Tampa Scale for Kinesiophobias: 13-item adjusted version; WHYMPI, West Haven-Yale Multidimensional Pain Inventory.
}

Despite these study limitations, the findings of this critically appraised topic demonstrate and support a relationship between kinesiophobia and patient perception of disability in upper-extremity injuries. Previous research has concluded that kinesiophobia can result in diminished performance that can ultimately lead to functional decline due to disuse or decreased activity. ${ }^{13}$ The majority of research regarding the impact of kinesiophobia on quality of life, however, has been conducted in the lower-extremity. Previous research has reported that lower-extremity injuries had a greater impact on health-related quality of life compared with upper-extremity injuries. ${ }^{14}$ The study by Sluys et $\mathrm{al}^{14}$ also found that patients with upper-extremity injuries showed larger improvements following surgical intervention. The prevalence and severity of lower-extremity injuries could provide an explanation for the larger body of knowledge regarding the impact of kinesiophobia. However, the upper-extremity is also essential in performing tasks in activities of daily living. ${ }^{7}$ The findings in this critically appraised topic demonstrate that kinesiophobia impacts upper-extremity injuries similar to lower-extremity injuries. Dealing with a functional disability to this body region could still greatly impede quality of life. It is well cited that an injury can result in kinesiophobia, which gives rise to behaviors such as hypervigilance, 
anxiety, and safety-seeking tendencies. ${ }^{15}$ In addition, previous research states that these subsequent behaviors can cause even greater long-term consequences and can lead to the development of chronic injury. ${ }^{5,15}$ In the case of the upper-extremity injuries, specifically, it is of utmost importance to mitigate feelings of kinesiophobia in order to prevent any subsequent behaviors that could prolong the healing process and significantly negatively impact quality of life.

Patient perceptions of their condition is a very important component to measure in clinical practice. Kinesiophobia can occur on a sliding scale and should be integrated into the patient's evaluation to compare with the clinical presentation, perceived disability scales, and quality of life measurements. A combination of these measurements can help to direct treatment goals and interventions. Following an evaluation, if the clinician finds heightened levels of kinesiophobia, the clinician can encourage the use of psychosocial strategies and interventions to help overcome or alleviate kinesiophobia. Psychosocial strategies, such as goal setting, imagery, positive self-talk, and relaxation techniques have been supported in the literature as positive coping mechanisms throughout the rehabilitation process. ${ }^{1}$ In addition, recent research has encouraged conducting a biopsychosocial evaluation to better understand the essence of each patient's pain. ${ }^{16}$ The purpose of a biopsychosocial evaluation is to gain a holistic view of the patient's presentation, identify any impairments across health dimensions, and guide a holistic treatment plan, which can help the clinician promote pain neuroscience education. ${ }^{16,17}$ Educating the patient on their pain could be an effective treatment strategy. Jones et $\mathrm{al}^{17}$ found that patients who had pain education in addition to therapeutic exercise showed the greatest improvements in self-reported pain.

It is of utmost importance for clinicians to understand the relationship between kinesiophobia and injury and their impact on quality of life, as well as to integrate intervention strategies into their clinical practice. A study by Comachio et $\mathrm{al}^{18}$ reported a significant correlation between increased kinesiophobia and decreased physical and emotional quality of life, as well as quality of pain, in patients with chronic low back pain. Comprehension of this relationship can encourage clinicians to ask their patients about their thoughts regarding their injury, educate their patient about their pain, and implement psychosocial intervention strategies. Specifically, clinicians should incorporate kinesiophobia, disability, or quality of life scales, such as the TSK, Disability of Arm Shoulder and Hand and/or Shoulder Pain and Disability Index, and Short-Form Health Survey, into the treatment plan of a patient experiencing kinesiophobia. Moreover, clinicians can educate their patients about the Fear-Avoidance Model and the potential consequences of pain catastrophizing which can help their patients to interpret their pain as nonthreatening rather than catastrophic. Incorporating these principles from a psychologically informed standpoint can improve and inform the rehabilitation process and treatment decisions for any level of kinesiophobia. ${ }^{6}$ In addition, information from these principles can also direct patient education. When clinicians have a stronger knowledge of kinesiophobia and the psychological impact of upper-extremity injury, they can be more effective in helping the patient overcome psychological barriers associated with an upper-extremity injury. ${ }^{19}$ Previous research has found that over $94 \%$ of patients thought it was helpful when their health care professional addressed the psychological aspect of injury. ${ }^{19}$ Furthermore, many health professionals themselves believe that they need to address the psychological aspects of injury in order to improve the effectiveness of their work. ${ }^{1}$
In conclusion, there is a relationship between kinesiophobia and perceived disability in upper-extremity injuries. Clinicians should consider kinesiophobia as a contributing factor that can affect the patient's perceived disability of their upper-extremity injury. With the influence that kinesiophobia, catastrophic thinking, and so on, has on pain and disability perception, clinicians should evaluate and integrate a holistic approach to their treatment which includes addressing kinesiophobia with upper-extremity injuries.

\section{References}

1. Clement D, Granquist M, Arvinen-Barrow M. Psychosocial aspect of athletic injuries as perceived by athletic trainers. J Athl Train. 2013;48(4): 512-521. PubMed ID: 23724772 doi:10.4085/1062-6050-48.3.21

2. Linton S, Buer N, Vlaeyen J, Hellsing A. Are fear-avoidance beliefs related to the inception of an episode of back pain? A prospective study. Psychol Health. 2000;14(6):1051-1059. PubMed ID: 22175261 doi:10.1080/08870440008407366

3. Kellezi B, Coupland C, Morriss R, et al. The impact of psychological factors on recovery from injury: a multicentre cohort study. Soc Psychiatry Psychiatr Epidemiol. 2017;52(7):855-866. PubMed ID: 27803978 doi:10.1007/s00127-016-1299-z

4. De S, Vranceanu A, Ring D. Contribution of kinesophobia and catastrophic thinking to upper-extremity-specific disability. $J$ Bone Joint Surg Am. 2013;95(1):76-81. PubMed ID: 23283376 doi:10. 2106/JBJS.L.00064

5. Crombez G, Eccleston C, Van Damme S, Vlaeyen J, Karoly P. Fearavoidance model of chronic pain: the next generation. Clin J Pain. 2012;28(6):475-483. PubMed ID: 22673479 doi:10.1097/AJP. 0b013e3182385392

6. Hsu C, Meiercavhtol A, George S, Chmielewski T. Fear of reinjury in athletes. Sports Health. 2017;9(2):162-167. PubMed ID: 27590793 doi:10.1177/1941738116666813

7. Goldberg P, Zeppieri G, Bialosky J, et al. Kinesiophobia and its association with health-related quality of life across injury locations. Arch Phys Med Rehabil. 2018;99(1):43-48. PubMed ID: 28760572 doi:10.1016/j.apmr.2017.06.023

8. Lentz A, Barabas J, Day T, Bishop M, George S. The relationship of pain intensity, physical impairment, and pain-related fear to function in patients with shoulder pathology. J Orthop Sports Phys Ther. 2009;39(4):270-277. PubMed ID: 19346624 doi:10.2519/jospt. 2009.2879

9. Walker N, Thatcher J, Lavallee D. A preliminary development of the Re-Injury Anxiety Inventory (RIAI). Phys Ther Sport. 2010;11(1):2329. PubMed ID: 20129120 doi:10.1016/j.ptsp.2009.09.003

10. Feleus A, van Dalen T, Bierma-Zeinstra S, et al. Kinesiophobia in patients with non-traumatic arm, neck, and shoulder complaints: a prospective cohort study in general practice. BMC Musculoskelet Disord. 2007;8(1):117. PubMed ID: 18045457 doi:10.1186/1471-2474-8-117

11. Genoese F, Baez S, Hoch J. The association of fear-avoidance beliefs and self-reported knee function in patients with a knee injury: a critically appraised topic. Int J Athl Ther Train. 2018;23(5):187-191. doi:10.1123/ijatt.2017-0108

12. Ebell M, Siwek J, Weiss B. Strength of Recommendation Taxonomy (SORT): a patient-centered approach to grading evidence in the medical literature. J Am Board Fam Pract. 2004;17(1):59-67. PubMed ID: 15014055 doi:10.3122/jabfm.17.1.59

13. Larsson C, Hansson E, Sundquist K, Jakobsson U. Kinesiophobia and its relation to pain characteristics and cognitive affective variables in older adults with chronic pain. BMC Geriatr. 2016;16(1):128. PubMed ID: 27387557 doi:10.1186/s12877-12016-10302-12876 
14. Sluys K, Shults J, Richmond T. Health related quality of life and return to work after minor extremity injuries: a longitudinal study comparing upper versus lower extremity injuries. Injury. 2016;47(4):824-831. PubMed ID: 26965363 doi:10.1016/j.injury.2016.02.019

15. Leeuw M, Goossens M, Linton S, Crombez G, Boersma K, Vlaeyen J. The fear-avoidance model of musculoskeletal pain: current state of scientific evidence. J Behav Med. 2007;30(1):77-94. PubMed ID: 17180640 doi:10.1007/s10865-006-9085-0

16. Wijma A, van Wilgen C, Meeus M, Nijs J. Clinical biopsychosocial physiotherapy assessment of patients with chronic pain: the first step in pain neuroscience education. Physiother Theory Pract. 2016;32(5): 368-384. PubMed ID: 27351769 doi:10.1080/09593985.2016. 1194651
17. Jones K, Tocco E, Marshall A, McLeod T, Bacon C. Pain education with therapeutic exercise in chronic nonspecific low back pain rehabilitation: a critically appraised topic. J Sport Rehabil. 2020; 29(8):1204-1209. PubMed ID: 32106086 doi:10.1123/jsr.20190345

18. Comachio J, Magalhaes M, Silva A, Marques A. A cross-sectional study of associations between kinesiophobia, pain, disability, and quality of life in patients with chronic low back pain. Adv Rhematol. 2018;58(8):8. PubMed ID: 30657061 doi:10.1186/s42358-0180011-2

19. Russell H, Tracey J. What do injured athletes want from their health care professionals? Int J Athl Ther Train. 2011;16(5):18-21. doi:10. 1123/ijatt.16.5.18 